\title{
Small bowel perforation caused by applicator implantation in high-dose-rate interstitial brachytherapy for recurrent pelvic tumor: a case report
}

\author{
Koji Masui, MD', Hideya Yamazaki, MD, PhD', Gen Suzuki, MD, PhD', Daisuke Shimizu, MD, PhD', Tadashi Takenaka, RTT' \\ Yasuhiro Yamada, MD, PhD², Koji Okihara, MD, PhD², Yoshiaki Kuriu, MD, PhD ${ }^{3}$, Kei Yamada, MD, PhD' \\ 'Department of Radiology, Kyoto Prefectural University Graduate School of Medical Science, Kyoto, Japan, ${ }^{2}$ Department of Urology. Kyoto \\ Prefectural University Graduate School of Medical Science, Kyoto, Japan, ${ }^{3}$ Department of Surgery, Kyoto Prefectural University Graduate \\ School of Medical Science, Kyoto, Japan
}

\begin{abstract}
High-dose-rate interstitial brachytherapy (HDR-ISBT) is widely used for the treatment of pelvic tumors. However, there are a few reports on complications of applicator implantation in HDR-ISBT. We describe a case of small bowel perforation caused by applicator implantation in an 82-year-old male patient with recurrence tumor in the pelvis treated with HDR-ISBT. Eventually, the patient underwent laparotomy and partial intestinal resection. We recognized the site where the applicator was inserted into a part of the tumor on the mucosal surface. Pathological examination confirmed that the tumor had infiltrated the small intestine directly and that the infiltrated part had reached the submucosa.

This is the first published report about small bowel perforation caused by applicator implantation. In cases where intestinal infiltration of the tumor is suspected, HDR-ISBT should be performed with maximum caution.

J Contemp Brachytherapy 2020; 12, 2: 188-192 DOI: https://doi.org/10.5114/jcb.2020.93875
\end{abstract}

Key words: bowel perforation, pelvic tumor, high-dose-rate interstitial brachytherapy.

\section{Purpose}

High-dose-rate interstitial brachytherapy (HDR-ISBT) is widely used for the treatment of pelvic tumors, including prostate cancer $[1,2,3,4,5]$, uterine cervical cancer $[6,7]$, recurrent cancer in pelvic area, and anorectal cancer $[8,9,10,11,12]$.

Because HDR-ISBT requires applicator insertion, pelvic tumor treatment poses risks of bleeding, infection, or intestinal perforation. However, there are a few reports on complications caused by applicator implantation in HDR-ISBT [13]. Herein, we describe a case with recurrent bladder cancer in the pelvis who developed small bowel perforation during HDR-ISBT. To the best of our knowledge, this is the first report of small bowel perforation caused by applicator implantation in HDR-ISBT.

\section{Case presentation}

An 82-year-old man was diagnosed with recurrence of bladder cancer. He underwent radical cystectomy and ureterocutaneostomy for bladder cancer in 2007. Unfortunately, during the surgery, a rectal damage occurred, and he had to undergo temporal colostomy and multiple subsequent abdominal open surgeries for restoration. In 2015 , a recurrent tumor $(40 \times 42 \times 26 \mathrm{~mm})$ was detected on computed tomography (CT) on the right side of the rectum (Figure 1A, B). The tumor was strongly suspected to be directly infiltrating the rectum, but infiltration to the surrounding small bowel was not evident. Contrast-enhanced total body CT detected no other tumors except the abovementioned lesion. The patient refused chemotherapy and surgery because of his age. He also refused external beam radiotherapy, which required long treatment period. He eventually agreed to undergo ISBT with radical intention because of its short treatment period and high local effect.

After administration of epidural anesthesia, 11 applicators (ProGuide Sharp Needle; Nucletron, ELEKTA $\mathrm{AB}$, Stockholm, Sweden) were percutaneously inserted into the tumor under transrectal ultrasound (TRUS) 

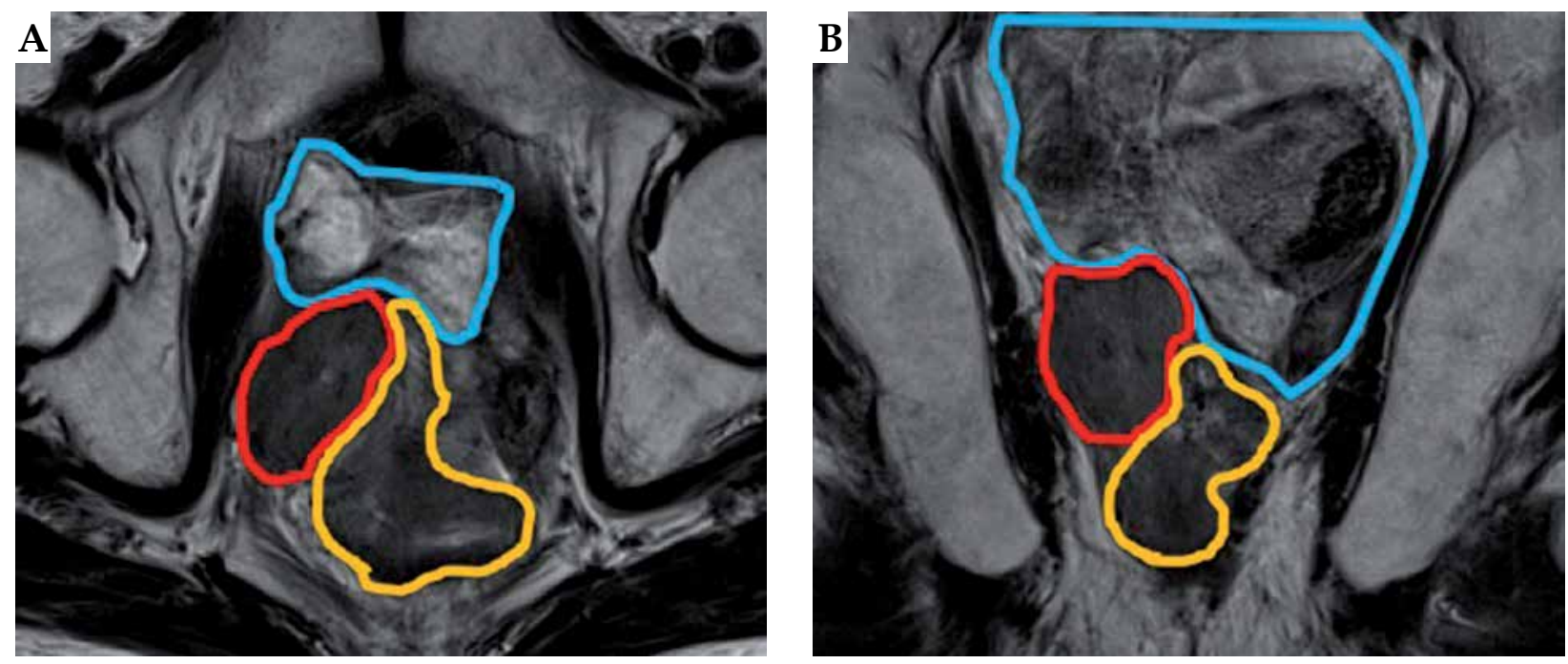

Fig. 1. Magnetic resonance image of the recurrent tumor. A) Axial image; B) Coronal image. The red line depicts the tumor, the orange line depicts the rectum, and the blue line depicts the small bowel

guidance (ALOKA ProSound Alpha 7, Hitachi ALOKA Medical, Tokyo, Japan). Applicator insertion was completed without any trouble. Subsequently, we performed
$\mathrm{CT}$ and magnetic resonance (MR) image, and radiation therapy was planned (Figure 2A, B). In the treatment planning system (Oncentra ${ }^{\circledR}$ brachy; Nucletron, ELEKTA
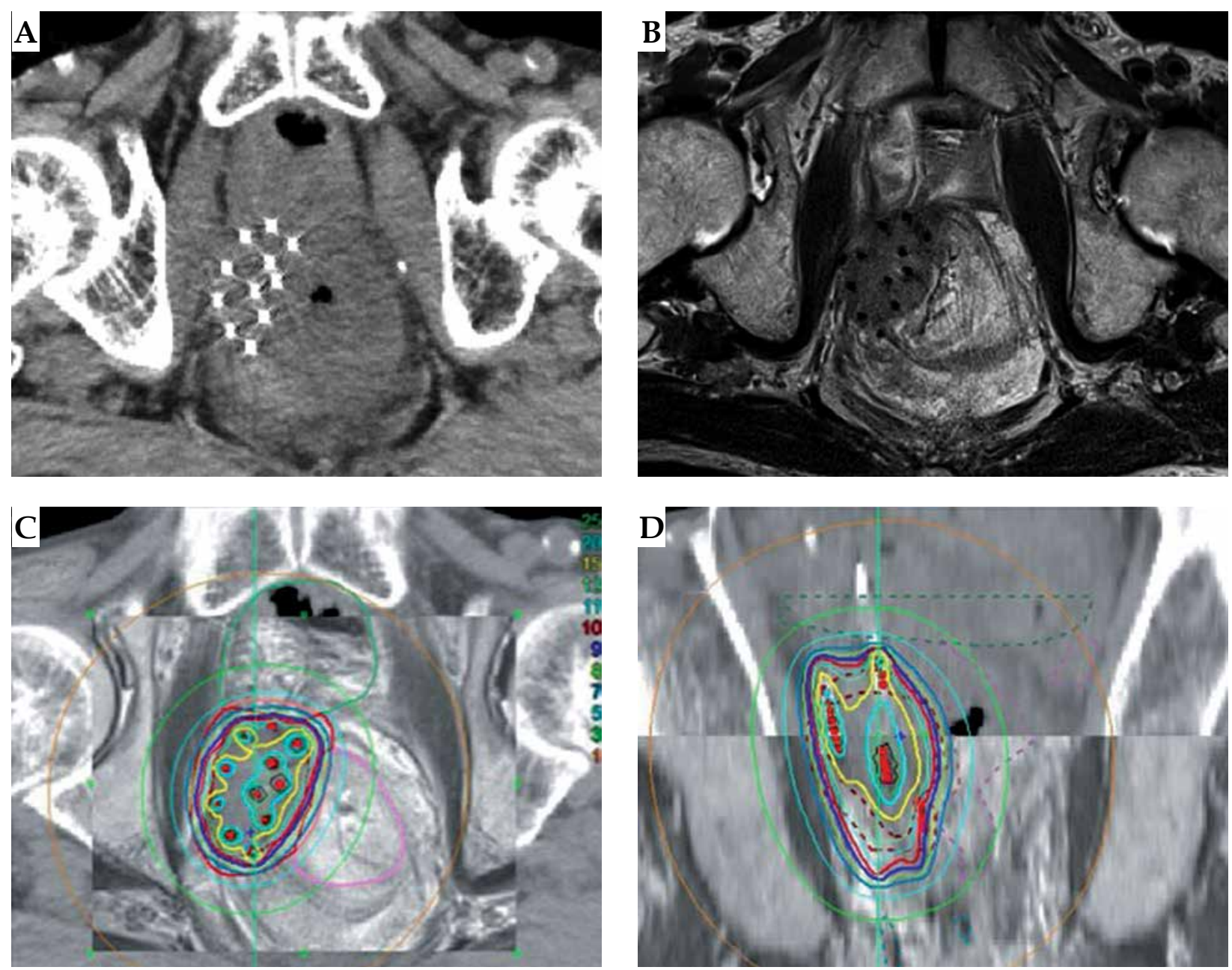

Fig. 2. Treatment planning. A) Computed tomography image; B) Magnetic resonance image; C) Treatment planning (axial image); D) Treatment planning (coronal image) 

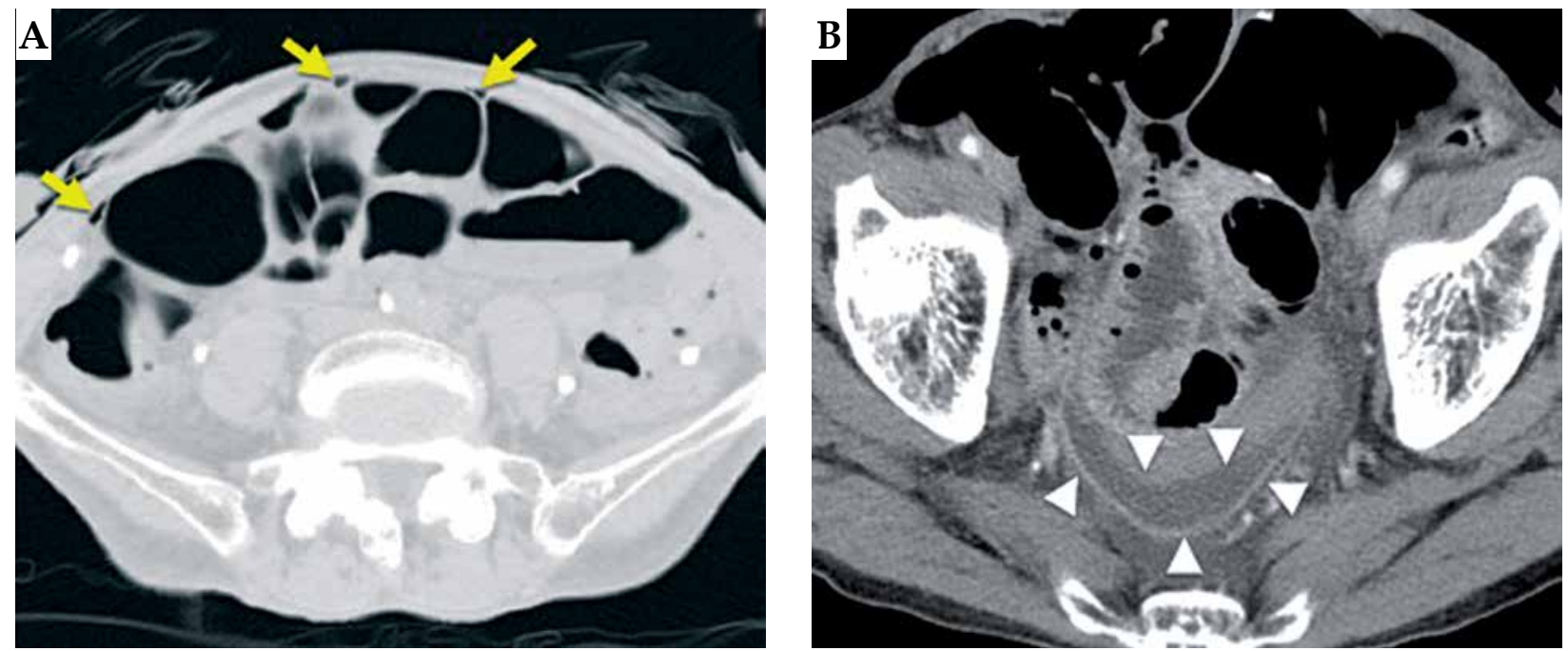

Fig. 3. Computed tomography images one day after treatment initiation. A) The yellow arrows depict free air; B) The white arrowheads depict an abscess (a fluid collection surrounded by an enhanced ring)

$\mathrm{AB}$, Stockholm, Sweden), we delineated the gross tumor volume (GTV) using the planning system function of CT/ MR image registration (Figure 2C, D), and 6 Gy per fractionated dose was prescribed for $98 \%$ of the GTV. Minimum doses to the most exposed $2.0 \mathrm{cc}\left(\mathrm{D}_{2 \mathrm{cc}}\right)$ of the rectum and the small bowel were 4.3 Gy and 4 Gy per fraction, respectively. HDR-ISBT of 54 Gy was planned to be administered in nine fractions for five days (twice daily with six hours interval), using a ${ }^{192}$ Ir remote after-loading system (MicroSelectron v3 ${ }^{\circledR}$ HDR, Nucletron, ELEKTA AB, Stockholm, Sweden).

HDR-ISBT was started as planned. The patient had no clinical symptoms during or after the initial treatment. On the second day of treatment, however, his laboratory data showed an increased white blood cells count $(19,200 / \mu \mathrm{l})$ and C-reactive protein level $(10.5 \mathrm{mg} / \mathrm{dl})$ after two fractions of treatment and the total dose of 12 Gy. Although the patient had no clinical symptoms, we performed CT to detect any adverse events. Free air and a fluid collection surrounded by an enhanced ring were noted and imaging diagnosis of an abscess at pelvic floor was made (Figure 3A, B); however, we could not identify the exact location of intestinal perforation. We stopped the treatment and removed the applicators. Fasting management was started and the antibiotic treatment was changed from flomoxef ( $3 \mathrm{~g} /$ day $)$ to meropenem ( $1.5 \mathrm{~g} /$ day). The following day, signs of local peritoneal irritation were noted and we decided to perform laparotomy.

Intraoperatively, a small amount of bleeding and pus was observed at the pelvic floor (Figure 4A) and the tumor adhered to the small intestine. Even then we could not directly pinpoint the perforation site. After careful irrigation of the abdominal cavity, a part of the small bowel that was attached to the tumor was removed.

When we cut opened the resected small bowel, we recognized the site where the applicator was inserted into a part of the tumor on the mucosal surface (Figure 4B). Finally, pathological examination confirmed that the tumor had infiltrated the small intestine directly and that the infiltrated part had reached the submucosa (Figure 4C, D).

The postoperative course was uneventful. The patient started oral ingestion a week later and was discharged from the hospital two weeks after admission (Figure 5). He was followed up without further treatment, and there had been no tumor growth nor any other complications. The patient died 20 months later of systemic metastases from his bladder cancer.

\section{Discussion}

The method of applicator insertion under TRUS guidance has been very popular in the treatment of prostate cancers [1,2,3,4,5]. After Stock et al. first reported on the treatment of cervical cancer [14], the same method has been widely used in gynecological malignancies and some other recurrent tumors in the pelvic cavity $[6,7,8,9,10,12,13]$.

ISBT for pelvic site tumors poses risks of bleeding, infection, and intestinal perforation. However, there has been only one report of complications caused by applicator implantation by Sharma et al. [13]. They also used TRUS guidance and observed a perioperative complication rate of $2.5 \%$ in the form of hematuria. They investigated the locations of the applicators using CT images obtained for treatment planning and they did not encounter any case with perforation. Thus, they suggested that TRUS assists in avoiding the needle injury/perforation of the pelvic structures, thereby reducing the risk of perioperative complications.

In our hospital, we have treated 82 patients who had tumors at pelvic sites with HDR-ISBT under TRUS guidance between September 2014 and August 2019. We retrospectively investigated whether we have inserted any of the applicators directly into the intestine, using MR images obtained for treatment planning. We had inserted a total of 1,014 applicators, and there were only two $(0.2 \%)$ that appeared to be inserted into the intestine. Both 

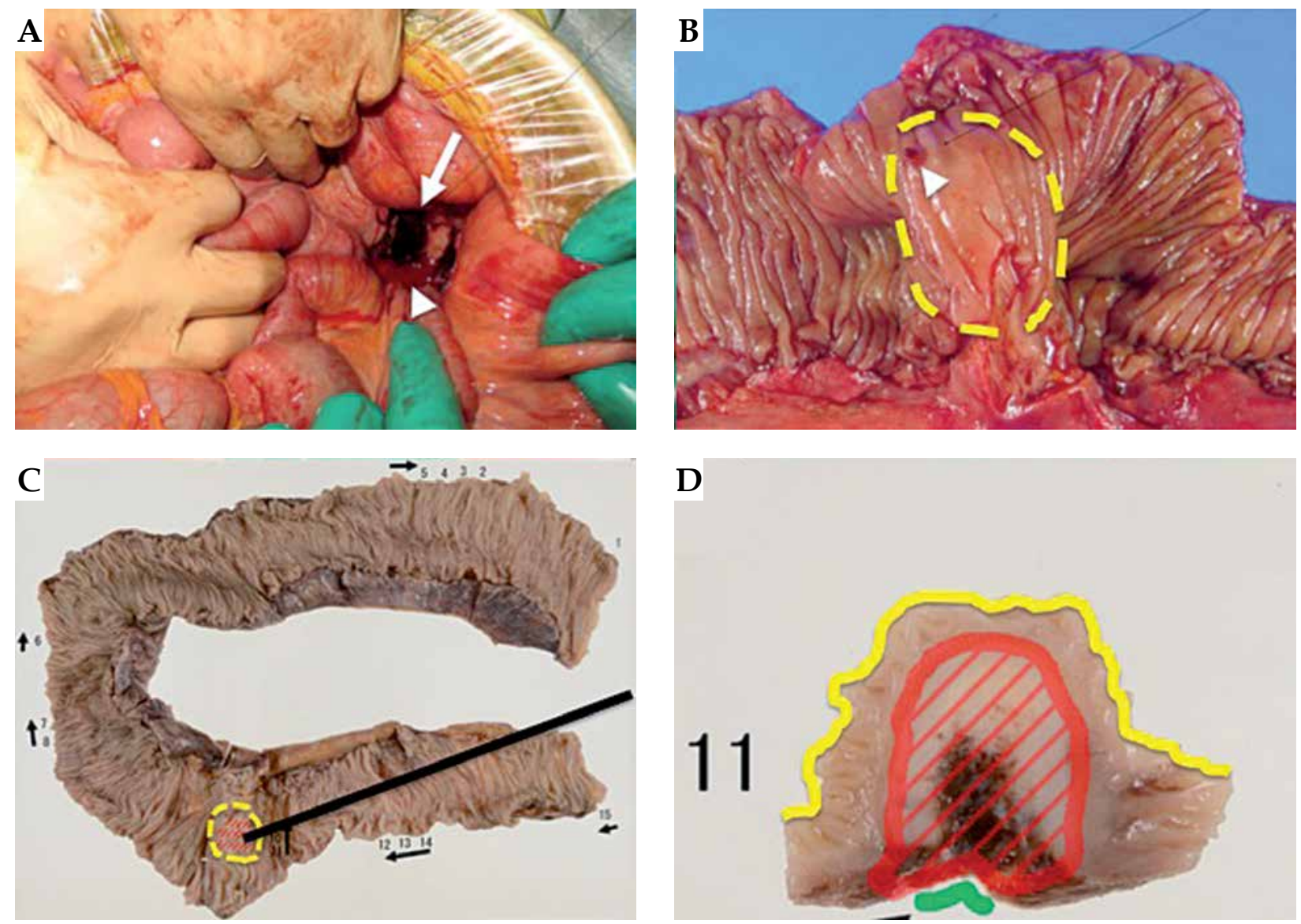

Fig. 4. Perioperative imaging. A) Intraoperative image. The white arrow depicts the pus, the white arrowhead depicts hemorrhage; B) Resected small bowel after surgery. The white arrowhead depicts the applicator pathway; C) Resected small bowel after formalin fixation. The yellow dotted line depicts the tumor; D) Resected small bowel after formalin fixation (larger scale). The red line depicts the tumor and the yellow line depicts the mucosal surface

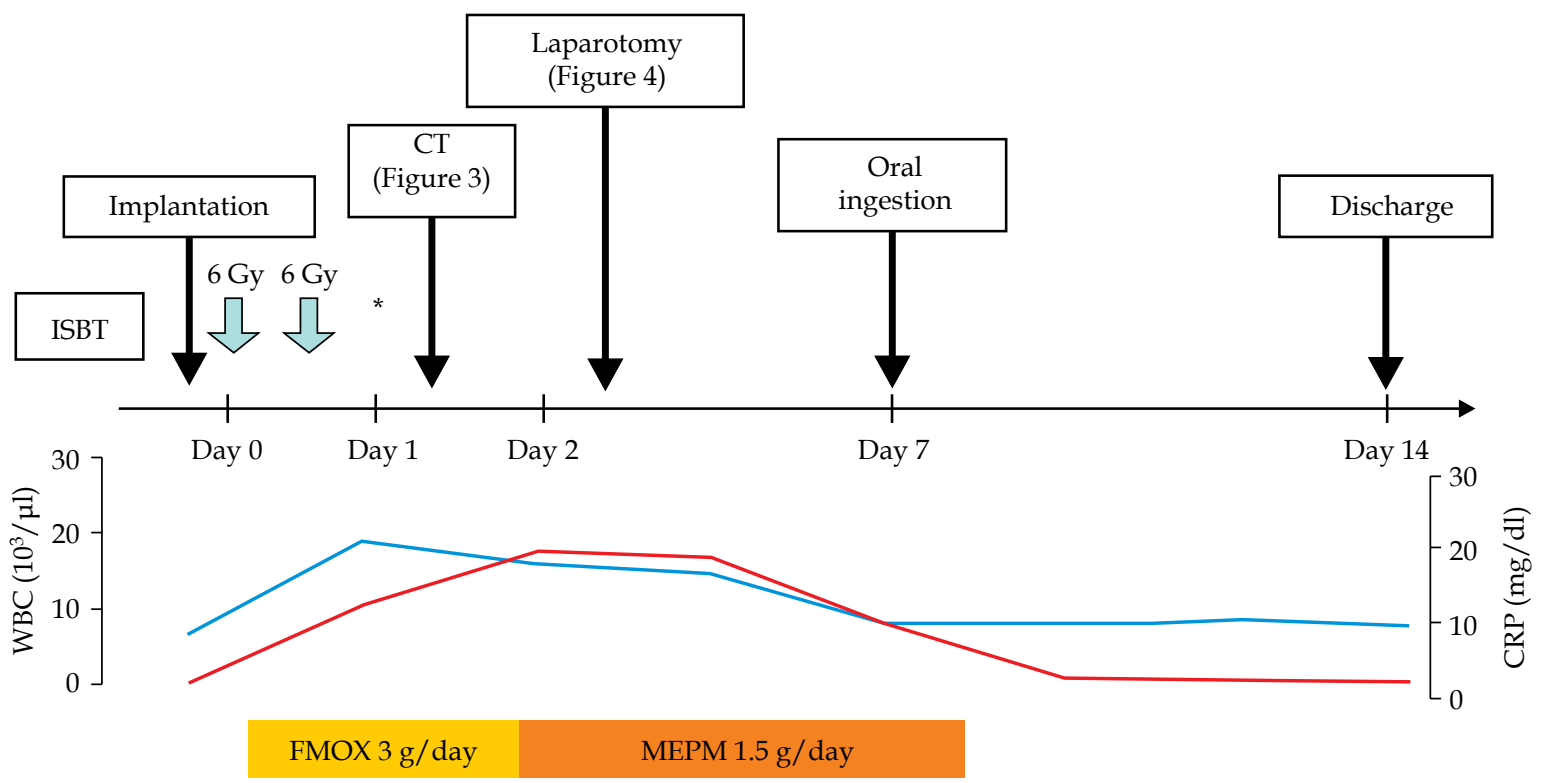

Fig. 5. Patient's treatment course

*treatment discontinuation and applicator removal; ISBT - interstitial brachytherapy, CT - computed tomography, WBC - white blood cells (blue line), $C R P$ - C-reactive protein (red line), FMOX - flomoxef, MEPM - meropenem 
applicators were observed in the patient of this report. None of the other patients had tumor clearly suspected of intestinal infiltration on MR images.

As mentioned above, MR images confirmed that two applicators were inserted into small intestine. In actual, however, only one applicator was found to penetrate the small intestine. The intestinal perforation might have occurred because the intestine penetrated by one applicator had an abnormal mucosa infiltrated directly by the tumor, while the intestine penetrated by another applicator had a normal mucosa, so the perforation in the second case might have not occurred.

The small bowel perforation may have been caused by mucosal insertion and mucosal irradiation. Because the perforation was observed soon after the start of BT, the treatment plan was made so that the dose to small bowel was reduced as much as possible, since the influence of mucosal insertion was considered significant.

In most $\mathrm{c}$ ases of postoperative tumor recurrence in the pelvis, there are intestines at the cranial side of tumors. If there is any possibility that the tumor had infiltrated the intestine, we recommend laparoscopy to be used $[15,16,17]$.

\section{Conclusions}

This is the first case report of small bowel perforation caused by applicator implantation in HDR-ISBT. In cases where intestinal infiltration of the tumor is suspected, HDR-ISBT should be performed with maximum caution. stitial brachytherapy for recurrent uterine carcinoma after curative surgery. J Radiat Res 2011; 52: 329-334.

10. Yoshida K, Yamazaki H, Kotsuma T et al. Treatment results of image-guided high-dose-rate interstitial brachytherapy for pelvic recurrence of uterine cancer. Brachytherapy 2015; 14: $440-448$

11. Yamazaki $H$, Inoue $T$, Ikeda $H$ et al. High-dose-rate remote afterloading intestinal radiotherapy employing the template technique for recurrent cancer in the pelvic area. Strahlenther Onkol 1993; 169: 481-485.

12. Bishop AJ, Gupta S, Cunningham MG et al. Interstitial brachytherapy for the treatment of locally recurrent anorectal cancer. Ann Surg Oncol 2015; 22 Suppl 3: S596-602.

13. Sharma DN, Rath GK, Thulkar S et al. Use of transrectal ultrasound for high dose rate interstitial brachytherapy for patients of carcinoma of uterine cervix. J Gynecol Oncol 2010; 21: 12-17.

14. Stock RG, Chan K, Terk M et al. A new technique for performing Syed-Neblett template interstitial implants for gynecologic malignancies using transrectal-ultrasound guidance. Int J Radiat Oncol Biol Phys 1997; 37: 819-825.

15. Vasilev SA, Vora N, Lee Y. Laparoscopically assisted transperineal interstitial brachytherapy with omental flap for locally recurrent endometrial cancer. J Laparoendosc Surg 1995; 5: 393-397.

16. Choi JC, Ingenito AC, Nanda RK et al. Potential decreased morbidity of interstitial brachytherapy for gynecologic malignancies using laparoscopy: A pilot study. Gynecol Oncol 1999; 73: 210-215

17. Nap-van Klinken A, Bus SJ, Janssen TG et al. Interstitial brachytherapy for bladder cancer with the aid of laparoscopy. J Contemp Brachytherapy 2014; 6: 313-317.

\section{Disclosure}

The authors report no conflict of interest.

\section{References}

1. Hoskin P. High dose rate brachytherapy for prostate cancer. Cancer Radiother 2008; 12: 512-514.

2. Yoshioka Y, Konishi K, Sumida I et al. Monotherapeutic high-dose-rate brachytherapy for prostate cancer: five-year results of an extreme hypofractionation regimen with 54 Gy in nine fractions. Int J Radiat Oncol Biol Phys 2011; 80: 469-475.

3. Zamboglou N, Tselis N, Baltas D et al. High-dose-rate interstitial brachytherapy as monotherapy for clinically localized prostate cancer: treatment evolution and mature results. Int J Radiat Oncol Biol Phys 2013; 85: 672-678.

4. Yoshida K, Yamazaki H, Takenaka T et al. High-dose-rate interstitial brachytherapy in combination with androgen deprivation therapy for prostate cancer: are high-riskpatients good candidates? Strahlenther Onkol 2014; 190: 1015-1020.

5. Tselis N, Hoskin P, Baltas D et al. High dose rate brachytherapy as monotherapy for localised prostate cancer: Review of the current status. Clin Oncol (R Coll Radiol) 2017; 29: 401-411.

6. Beriwal S, Bhatnagar A, Heron DE et al. High-dose-rate interstitial brachytherapy for gynecologic malignancies. Brachytherapy 2006; 5: 218-222.

7. Yoshida K, Yamazaki H, Takenaka T et al. Preliminary results of MRI-assisted high-dose-rate interstitial brachytherapy for uterine cervical cancer. Brachytherapy 2015; 14: 1-8.

8. Beriwal S, Heron DE, Mogus $R$ et al. High-dose rate brachytherapy (HDRB) for primary or recurrent cancer in the vagina. Radiat Oncol 2008; 3: 7.

9. Kotsuma T, Yoshida K, Yamazaki H et al. Preliminary results of magnetic resonance imaging-aided high-dose-rate inter- 